Artículo de posesión

\title{
La interdisciplinariedad en la ciencia del rayo
}

\author{
Horacio Torres-Sánchez \\ Profesor Emérito Universidad Nacional de Colombia \\ Artículo de posesión para el ingreso como miembro de número a la \\ Academia Colombiana de Ciencias Exactas, Físicas y Naturales el 26 de julio de 2017
}

\begin{abstract}
Resumen
Este artículo sobre la investigación del fenómeno del rayo ha sido elaborado para la posesión como Miembro de Número de la Academia Colombina de Ciencias Exactas, Físicas y Naturales. El fenómeno natural ha sido investigado sistemáticamente en Colombia desde hace aproximadamente cuatro décadas motivado por la curiosidad científica para comprender la alta mortalidad de sistemas, equipos y de personas. Con base en el desarrollo de una hipótesis de investigación se fueron confrontando sus resultados con la realidad concreta, mediante el método científico inductivo y la categoría conceptual de un trabajo interdisciplinario con vínculos dialecticos y la intersección e integración de saberes como la física, las matemáticas, la química, la biología, la historia, la literatura y la psicología social. Con los resultados de investigación se ha logrado aportar nuevo conocimiento del fenómeno en el concierto mundial con libros, artículos científicos, patentes, innovaciones tecnológicas para aplicaciones en protección contra rayos, normas y reglamentos técnicos y apropiación social de la ciencia. Este articulo presenta los logros científicos y tecnológicos realizados en Colombia en el campo de las descargas eléctricas atmosféricas, y propone, basado en las condiciones específicas intertropicales, los focos de investigación de interés para el país en el futuro. (C) 2017. Acad. Colomb. Cienc. Ex. Fis. Nat.
\end{abstract}

Palabras clave: Rayos; Interdisciplinariedad; Método científico inductivo.

The interdisciplinarity of lightning

\begin{abstract}
This paper on the research of the lightning phenomenon has been elaborated for the author's possession as a Member of Number of the Colombian Academy of Exact, Physical and Natural Sciences. This phenomenon has been systematically investigated in Colombia in the last four decades or so, motivated by the scientific curiosity to understand the high incidence of damage to systems and equipment, as well as mortality of people. Based on the development of a research hypothesis, the results were compared with concrete reality by using the scientific inductive method and the interdisciplinary work with dialectical links and the intersection and integration of knowledge from Physics, Mathematics, Chemistry, Biology, History, Literature and Social Psychology. The results have contributed with new knowledge in the world concert with research books, papers, patents and technological innovations for applications in lightning protection, technical standards, technical regulations and social appropriation of the science. This article presents the scientific and technological achievements made in Colombia in the field of atmospheric electric discharges, and proposes, based on specific intertropical conditions, the research focus of interest to the country in the future. (C) 2017. Acad. Colomb. Cienc. Ex. Fis. Nat.
\end{abstract}

Key words: Lightning, science; Interdisciplinary; Inductive scientific method.

\section{Introducción}

El fenómeno del rayo ha sido investigado sistemáticamente en Colombia desde hace aproximadamente cuatro décadas con la creación de un grupo de investigación, motivado por la curiosidad científica del fenómeno, para comprender la alta mortalidad de sistemas, equipos y de personas y con el objetivo de llevar a cabo un proceso de apropiación, construcción y creación autónoma de conocimiento.

El propósito fue avanzar tanto en el conocimiento como en la solución a la problemática de los rayos, lo cual ha generado innovaciones tecnológicas, reconocimientos, valiosos resultados visibles y comunicables que han sido susceptibles de contrastación académica internacional y de validez social. Consecuentemente, sus productos se han proyectado positivamente hacia la productividad y competitividad del país.

\footnotetext{
Correspondencia:

Horacio Torres-Sánchez, htorress@unal.edu.co

Recibido: 04 de mayo de 2017

Aceptado: 14 de junio de 2017
} 
Tras una primera etapa de mediciones, análisis y clasificación se logró postular una hipótesis para lograr una comprensión científica amplia y profunda del origen, características y variaciones del fenómeno del rayo.

Con base en el desarrollo de la hipótesis planteada se fueron confrontando sus resultados con la realidad concreta. Sin perder de vista que en el método científico no existen verdades absolutas, sino certezas temporales y los resultados tienen que ajustarse continuamente, pues el fenómeno del rayo, al igual que todos los fenómenos de la naturaleza, se encuentra en permanente cambio (Torres, 2015).

En el proceso realizado de ir de lo particular a lo general y luego regresar a lo particular se trabajó, fundamentalmente, el método inductivo, científicamente aceptado por la comunidad académica internacional. Una forma de llevar a cabo el método inductivo fue proponer, mediante diversas observaciones de los sucesos, una conclusión que resultara general para todos los eventos de la misma clase.

\section{La interdisciplinariedad}

La categoría conceptual del trabajo interdisciplinario, entendida como una forma de conocimiento aplicado que se produce en la intersección de los saberes ha sido una percepción más amplia de comprender y abordar el fenómeno del rayo.

En el proceso de investigación científica se hizo necesario acudir al aporte de disciplinas diferentes a la física y las matemáticas, que no habían sido consideradas inicialmente en el análisis del fenómeno, para comprenderlo y abordarlo de una forma integral más objetiva: con los aportes de la química para comprender los beneficios del rayo en la producción de ozono y el proceso de fijación química que el rayo ejerce para la combinación del nitrógeno con oxígeno o hidrógeno; la biología para comprender los efectos del rayo en el cuerpo humano; la historia y la literatura para comprender las explicaciones del fenómeno a través de otros lenguajes como el mítico, el literario y el religioso que permiten ver la evolución y los avances en el entendimiento del fenómeno a través de las diferentes culturas y la psicología social para comprender el comportamiento de los seres humanos en grupo en la prevención de los efectos negativos del rayo.

En la medida en que se continúe profundizando en el conocimiento del fenómeno se necesitará de otras disciplinas con la exitosa metodología lograda.

Para realizar la labor interdisciplinaria no se precisó que todos los académicos asesores estuvieran en el mismo grupo de investigación, sino que participaron activamente con una visión distinta desde su saber, logrando integrar su conocimiento con el conocimiento nuestro alrededor del fenómeno del rayo para solucionar problemáticas particulares.

Se tuvieron claros los límites del saber de cada uno y se respetaron los campos de conocimiento, conociendo lo propio, precisado no sólo por lo que se sabía sino por lo que se ignoraba.

\section{Planteamiento de la hipótesis de investigación}

La hipótesis de investigación sobre la variación espacial y temporal en los parámetros del rayo ha estado siempre presente en todos los proyectos académicos y en las tesis de pregrado, maestría y doctorado que se han realizado y dirigido en el grupo Programa de Investigación en Adquisición y Análisis de Señales - PAAS, del departamento de Ingeniería Eléctrica de la Universidad Nacional de Colombia, Una de las líneas de investigación del Programa PAAS ha sido el de las “Descargas eléctricas atmosféricas en zona tropical” y fue presentada en el trabajo de promoción a Profesor titular (Torres, 1998) en la Universidad Nacional de Colombia y posteriormente en el libro El Rayo (Torres, 2002).

La hipótesis se fundamentó en los principios científicos planteados por el físico inglés Wilson (1920) y Whipple (1929), sobre el circuito eléctrico global y la contribución dominante, por una superposición de efectos, de las tres mayores zonas de convección profunda tropical del planeta: Sur América tropical, centro de África y el continente marítimo (Sur este de Asia y Australia).

Si bien las zonas de convección profunda tropical fueron identificadas al principio del siglo XX como de alta actividad eléctrica atmosférica, en el mundo la mayoría de la información disponible sobre las características y magnitudes de los rayos estaban basadas en estudios llevados a cabo en zonas semitropicales o templadas, zonas con clima caracterizado por cambios bien definidos en las estaciones. El rango representativo de latitudes asociado con este tipo de clima se extiende desde los $25^{\circ}$ de latitud $\mathrm{N}$ o $\mathrm{S}$ hasta los $50^{\circ}$ de latitud $\mathrm{N}$ o $\mathrm{S}$, pero escasos en zonas tropicales.

Con base en los principios de Wilson y Whipple se inició el trabajo para la comprobación de la hipótesis tomando las primeras estimaciones que se hicieron del parámetro nivel ceráunico en Colombia en 1982 y posteriormente con mediciones, análisis matemático y revisión bibliográfica de los otros parámetros del rayo. El nivel ceráunico es un parámetro sobre la actividad de rayos en una región determinada y se mide en número de días de tormenta al año. Un día de tormenta se define, para este parámetro, como aquel en el cual es oído por lo menos un trueno.

Más de quince parámetros científicamente mensurables han sido establecidos durante el estudio sistemático de la física del rayo. La escala de órdenes de magnitud espacial va desde los fenómenos atómicos que inician la electrificación de la nube de tormenta en una magnitud de $10^{-10}$ metros, hasta el movimiento del aire de la nube de tormenta, que completa el proceso de carga, en una escala de decenas o centenas de kilómetros (Torres, 2015).

Con base en el proceso de desarrollar la hipótesis de investigación planteada, hoy en día sabemos, por ejemplo, que más de 2000 tormentas están activas alrededor del globo terráqueo en un momento dado, produciendo aproximadamente 100 rayos por segundo, que están más concentrados en la zona tropical terrestre que en las zonas templadas del planeta, como se muestra en las mediciones satelitales y 
en las mediciones terrestres que hemos realizado no solo en Colombia sino en Brasil o en Cuba. Y sus parámetros como la corriente de retorno del rayo, el nivel ceráunico, la densidad de rayos a tierra, la energía o su polaridad difieren estadísticamente a los medidos en la zona templada.

La figura 1 presenta un ejemplo de la variación espacial y temporal sobre el comportamiento del nivel ceráunico en Bogotá, Colombia y La Habana, Cuba de una base de datos multianual, (Torres, 2002).

El comportamiento bimodal en la ciudad de Bogotá, Colombia y mono modal en la ciudad de La Habana, Cuba, están directamente relacionados con el desplazamiento de la zona de confluencia intertropical, comportamientos que son totalmente diferentes en latitudes templadas (Torres, 2015).

La figura 2, (adaptada de Torres 2015) presenta otro ejemplo comparativo del parámetro nivel ceráunico multianual global para países en latitudes templadas (Ejemplo, Estados Unidos y Francia) y países tropicales (Cuba, Colombia y Brasil), donde el valor máximo multianual en Francia es de 36, mientras en Colombia como país tropical puede llegar a más de 200.

La figura 3 compara el parámetro probabilístico corriente de retorno de rayo adoptado por los países de Europa, Norteamérica y Asia agrupados en el comité CIGRE, el cual se
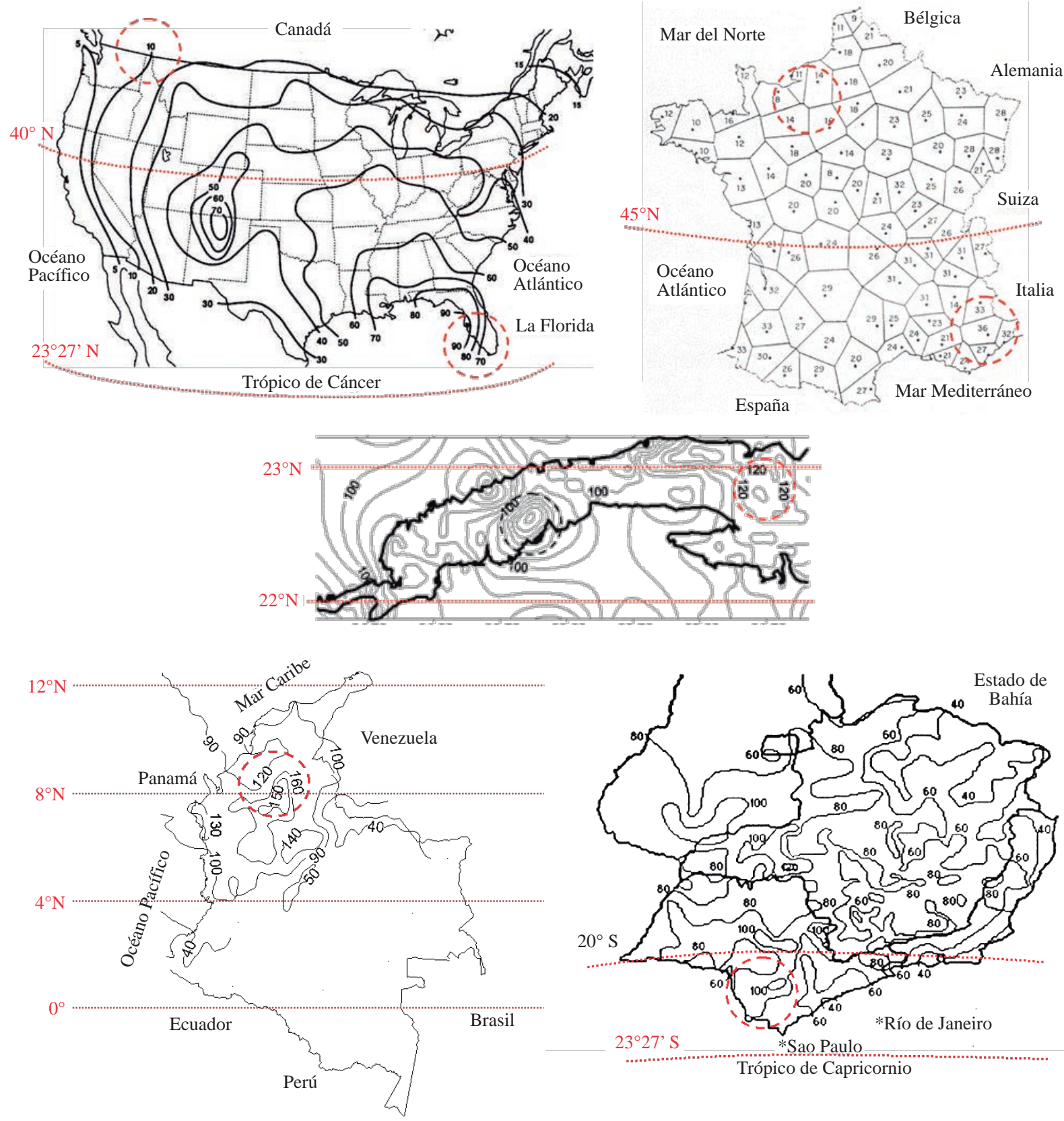

Figura 1. Variación mensual del Nivel Ceráunico en Bogotá, Colombia y La Habana, Cuba. 


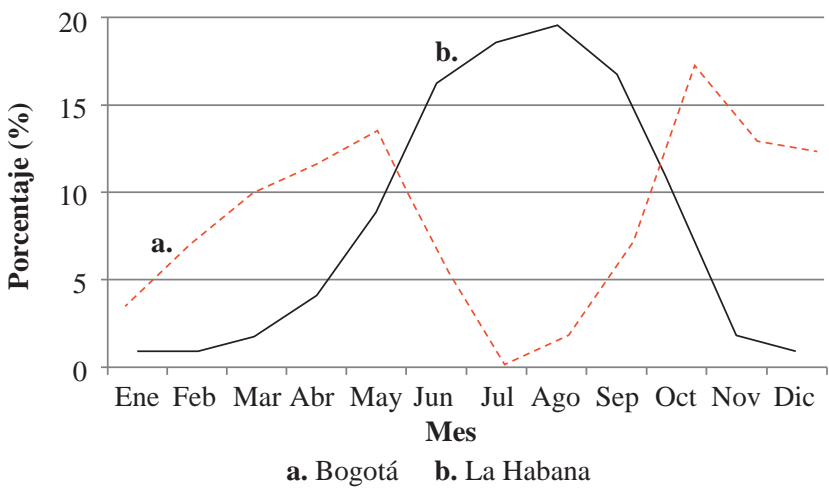

Figura 2. Índice Nivel Ceráunico multianual en diferentes países del mundo

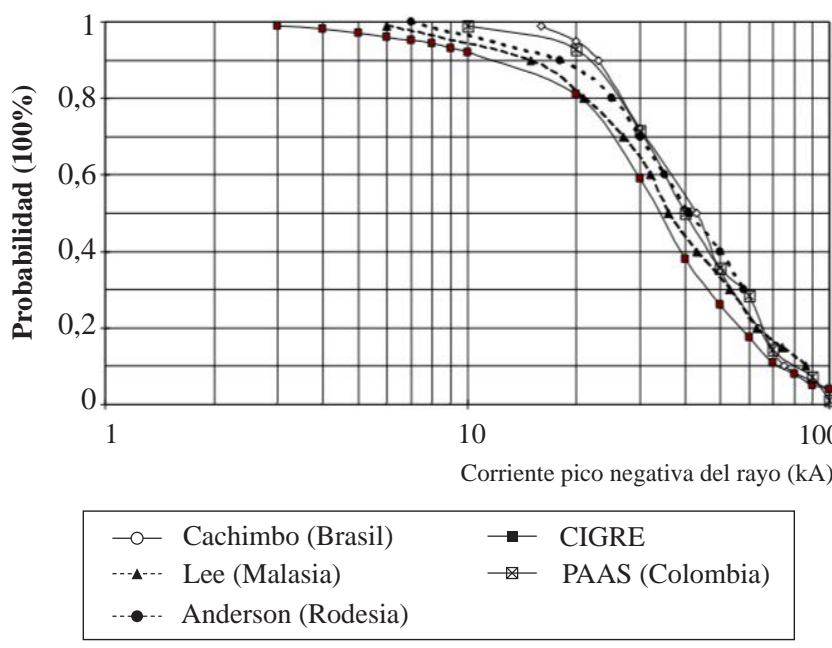

Figura 3. Índice probabilístico multianual de la Corriente de Retorno del Rayo para la mayoría de países del mundo (CIGRE) y países tropicales.

basa en las mediciones realizadas por Berger en el monte San Salvatore (Suiza) en la década de 1950 y reportadas subsecuentemente (Berger, 1975), con las más recientes, realizadas con equipos tecnológicamente más avanzados, en países tropicales como Brasil (Visacro, 2004), Rodesia (Anderson, 1980), Malasia (Lee, 1979) y Colombia (Torres, 1996). Mientras las mediciones de Berger conducen a una mediana multianual de $30 \mathrm{kA}$, las tropicales dan como resultado $45 \mathrm{kA}$ (Torres, 2015). Algunas de estas mediciones se hicieron de forma indirecta, utilizando sistemas de detección remota de rayos y, en consecuencia, los valores exactos pueden ser afectados por la validez de los modelos utilizados y por la eficiencia de detección de dichos sistemas.

Es así como hoy en día se tienen curvas de distribución de probabilidad para la corriente de retorno y la forma del impulso de la corriente de rayo, medidas en Europa, que se recomiendan tanto en la literatura especializada como en las normas internacionales, para ser utilizadas en diseño de protecciones contra rayos, diseño de aislamientos en máquinas eléctricas, diseño de apantallamientos en líneas de transmisión, para cualquier parte del mundo. Esta práctica sería equivalente a clasificar climatológicamente la región colombiana, según el científico alemán Köppen, como bosque húmedo tropical y, con base en ello, diseñar un equipo de aire acondicionado para un edificio en la ciudad de Bogotá, (Torres, 2002), (Torres, 2010).

Para medir los parámetros del rayo en zona tropical terrestre fue necesario hacer campañas de medición directa e indirecta con el diseño e instalación de una torre de medición directa de rayo en la zona de Samaná, Caldas y otras mediciones mediante medición por inducción. Estos parámetros fueron comparados con las mediciones similares en Brasil y otros países tropicales.

Todos estos parámetros fueron presentados y discutidos con la comunidad académica internacional (Torres, 2015) y luego fueron llevados a una Norma técnica colombiana (NTC 4552, 2008) (figura 4). Estos resultados producto de la investigación tienen implicaciones directas en la protección contra rayos de personas y bienes en general en la zona tropical, la cual, en definitiva, presenta magnitudes de los parámetros del rayo diferentes a las de latitudes templadas (Torres, 2015). Estos parámetros son, además de la corriente del rayo, su variación con respecto al tiempo (di/dt), su energía (W/R), su carga en culombios (Q), para polaridad positiva y negativa, entre otros.

\section{Física y matemáticas en el modelo del rayo}

Físicamente, el inicio del rayo se debe a la formación de penachos dentro de la nube, en la región inferior de carga negativa, a causa de los altos campos eléctricos allí presentes (de 0,1 a $1 \mathrm{MV} / \mathrm{m}$ ) (Torres, 2010), (Rakov, et.al., 2003), (MacGorman, et. al, 1998), (Cooray, 2003).

La propagación de estos penachos lleva a la recombinación de cargas en la base de la nube y a la formación de un canal eléctrico a partir del cual comienza a desarrollarse el líder del rayo. Aún no hay total claridad de la forma exacta como se producen estos penachos dentro de la nube, ya que existen teorías diversas que se basan en la precipitación de hidrometeoros, en la interacción de gotas de agua y en la creación de electrones energéticos a causa de rayos cósmicos (Torres, 2010), (MacGorman, et. al, 1998), (Cooray, 2003). Sin embargo, la mayor discusión y controversia se concentra en la forma como se propagan, con dos principales modelos conceptuales existentes: el unipolar y el bipolar.

Para la representación física del modelo de un rayo se parte de las leyes fundamentales del electromagnetismo que están basadas en observaciones experimentales que explican las variaciones en las propiedades físicas del fenómeno.

Estas leyes, conocidas como las leyes de Maxwell se expresan en términos de los cambios espaciales y temporales de las variables intervinientes. Es por ello que las ecuaciones diferenciales tienen importancia fundamental en las aplicaciones del fenómeno del rayo ya que los procesos físicos son modelados matemáticamente por estas ecuaciones. 


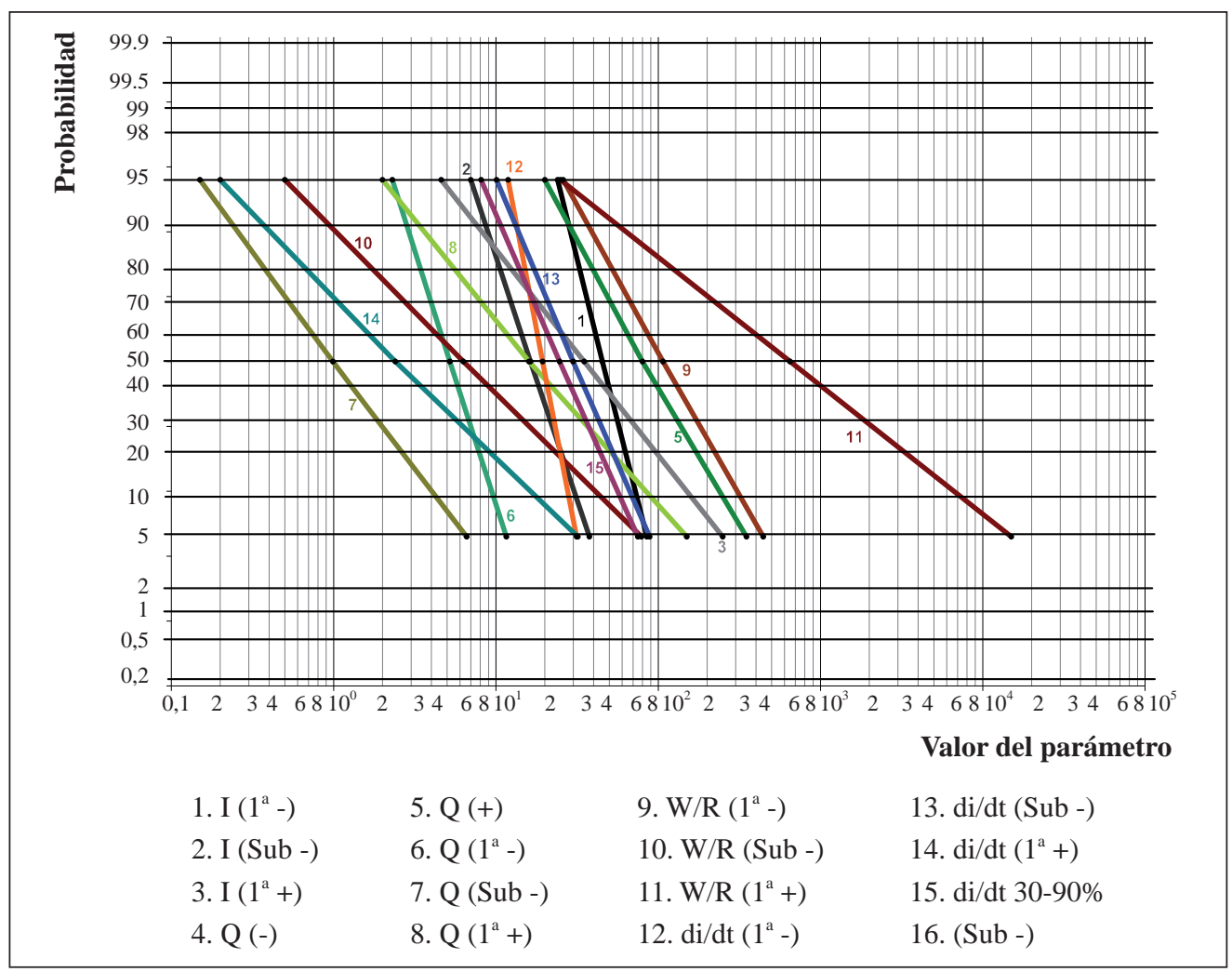

Figura 4. Parámetros probabilísticos del rayo, Norma Técnica Colombiana NTC 4552-1.

Hasta la fecha en que se comenzó el desarrollo de una tesis doctoral (Vargas, 2006) sobre el modelo físico y matemático del rayo se utilizaba, a nivel mundial, un modelo lineal. Fue necesario, entonces, resaltar que la influencia de la tortuosidad y las ramificaciones del líder descendente sobre el proceso de enlace y el desempeño de los sistemas de protección contra rayos, no había sido estudiada profundamente, Ver figura 5.

Algunos autores habían modelado la propagación y el camino del líder usando modelos probabilísticos basados en teoría de fractales (Petrov, 2003), (Agoris, et. al., 2004), (Lupo, 2000). Sin embargo, en estos trabajos no se hizo una estimación de la distribución de carga a lo largo del canal del líder y sus ramas y los canales obtenidos no se asemejaban a la geometría macroscópica real del rayo en aspectos como la longitud de pasos, cambios de dirección del líder y ángulos de ramificación.

Este trabajo fue presentado y discutido ante la comunidad académica internacional con la publicación de 2 artículos (Vargas, Torres, 2008). El modelo matemático integral desarrollado se basó en una aproximación electrostática, la cual se considera válida debido al avance escalonado del líder descendente y a su baja velocidad de propagación con respecto a la luz.

Las ecuaciones matemáticas no lineales fueron solucionadas mediante el método de simulación de cargas (MSC) y el método de cargas superficiales (MCS), derivado del MSC y que permite calcular potenciales y campos eléctricos a partir de una distribución de cargas superficiales dispuestas sobre el objeto.

Para solucionar el modelo, conocido como Bi-Líder, se construyó un sistema de ecuaciones en el cual el potencial en los puntos de contorno de las líneas de carga fueran la suma de las contribuciones de todas las líneas de carga, los centros de carga de la nube de tormenta (cargas esféricas) y sus imágenes. Permitió, así, considerar la tortuosidad y las ramificaciones aleatorias del líder descendente, con geometrías macroscópicas similares a las observadas en descargas naturales.

Además, se pudo estimar la distribución de carga a lo largo del canal del líder y sus ramas. Este modelo puede ser aplicado a una amplia variedad de estructuras en tierra encontradas en la realidad, mediante el modelamiento para estructuras esbeltas y robustas o complejas.

Complementariamente a la anterior tesis de doctorado se realizaron otras dos que aportaron al conocimiento universal del fenómeno del rayo y sus efectos en redes de energía eléctrica. El principal aspecto que se analizó en la tesis de doctorado (Herrera, 2006) fue la influencia que tiene la geometría del canal de la descarga eléctrica atmosférica en el cálculo tanto de campos electromagnéticos sobre un suelo de conductividad finita como en las tensiones inducidas que estos producen en líneas polifásicas aéreas de distribución. Se presentó, por primera vez a nivel mundial, este aporte 


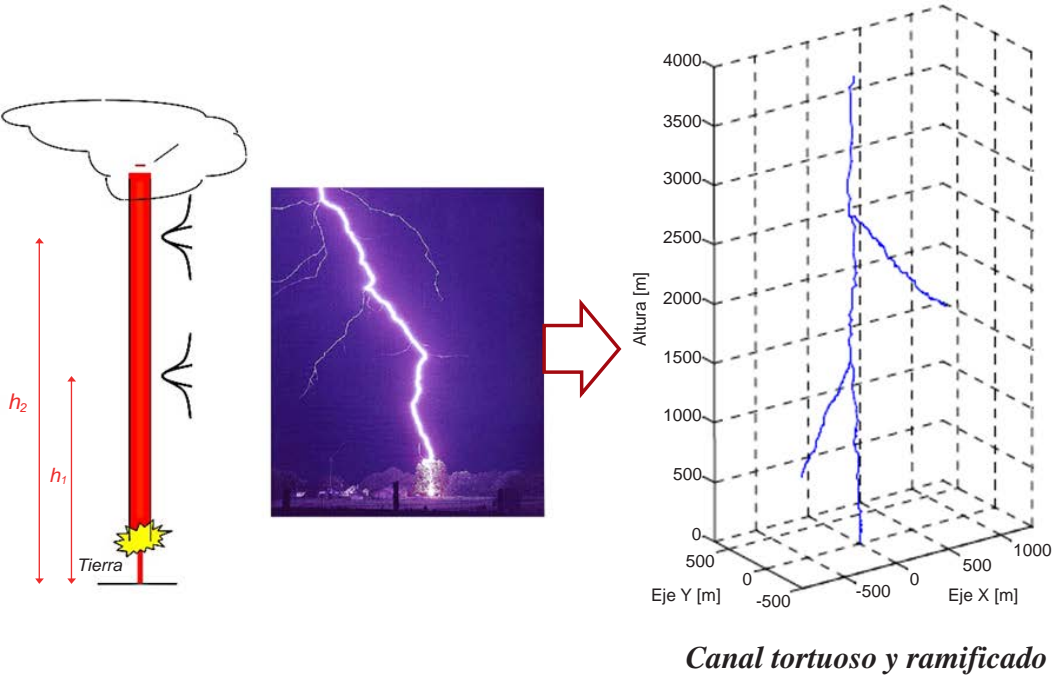

Figura 5. Modelo Bipolar: Análisis del modelo del rayo tortuoso y ramificado (Adaptada de Torres, H., 2015).

(Silveira, 2008). Y en (Pérez, 2006) los 3 principales aportes se realizaron en: a) descripción de los diferentes efectos de los parámetros del rayo y de la línea sobre las tensiones inducidas, b) identificación de los parámetros más influyentes en las tensiones inducidas y c) una ecuación sencilla para calcular tensiones inducidas máximas con errores menores que las ecuaciones existentes y así lograr aplicaciones para ingeniería eléctrica (Conti, 2010).

Mediante la tesis doctoral (Younes, 2006) se planteó un nuevo concepto que se denominó densidad de rayos difusa y surgió como uno de los principales aportes al conocimiento del fenómeno del rayo y sus aplicaciones en ingeniería, ya que es capaz de generar un concepto de densidad que se comporta más homogéneamente y para problemas prácticos de ingeniería, como la protección contra rayos y la evaluación de riesgo por rayos, puede llegar a ser más útil que el concepto tradicional de densidad de descargas a tierra (Torres, 2010).

Actualmente se realiza una tesis doctoral con información de una red instalada en los alrededores de la ciudad de Santa Marta, Colombia, dentro de una investigación financiada por la Agencia Aeroespacial Europea para caracterizar y proponer un modelo bidireccional de líderes para ser contrastados con los TGF (Terrestrial Gamma Ray Flashes).

\section{La biología y el fenómeno del rayo}

En la visión biológica sobre el fenómeno del rayo existen numerosos artículos científicos y de difusión científica respecto al peligro de los rayos para los seres humanos durante actividades al aire libre en caso de tormentas eléctricas.

Un rayo puede matar o causar daños al fluir corriente eléctrica por el cuerpo humano. Sin embargo, los flameos externos entre la cabeza y los pies, que se producen alrededor del cuerpo, pueden causar más daños como quemaduras que muerte.
La tasa de mortalidad por rayos varía entre 8 y 10\%, es decir que de cada 100 personas impactadas por rayo solamente entre 8 y 10 mueren. El paro cardiaco en el momento de la lesión es usualmente la causa de muerte; sin embargo, efectos secundarios, por ejemplo, golpes por caídas o asfixia pueden también ser mecanismos de muerte o lesión (Torres, Rojas, 2003).

Un impacto de rayo genera fundamentalmente una lesión neurológica que afecta los 3 componentes del sistema nervioso: central, autonómico y periférico. Un porcentaje alto de personas impactadas por la corriente de un rayo desarrollan alteraciones cardiacas y respiratorias que pueden ser pasajeras o tan duraderas que llevan a la muerte. En forma semejante a lo que experimentalmente se puede realizar en un laboratorio de electrofisiología estimulando células con pulsos de corriente, la energía de un rayo puede alterar los mecanismos celulares dependientes de las corrientes iónicas despolarizantes, originadas por el ion $\mathrm{Na}+\mathrm{o}$ las repolarizantes conducidas por el ión K+ (Torres, Rojas, 2003). Es posible inferir que, si el paso de la corriente del rayo coincide con el transcurso de la onda $\mathrm{T}$ de repolarización de la célula cardiaca, la fase de diástole o de relajación se alterará.

Estadísticamente, las quemaduras internas son raras en accidentes por rayos. De acuerdo con investigaciones internacionales, rara vez se ha encontrado mioglobina en la orina de las personas que han sufrido impactos por rayo. La mioglobina es una hemoproteína muscular, estructuralmente y funcionalmente muy parecida a la hemoglobina, cuya función es la de almacenar y transportar oxígeno dentro del tejido muscular. Cuando el músculo esquelético sufre algún daño, por ejemplo, quemaduras por corrientes de rayo, la mioglobina es liberada en el torrente sanguíneo y los riñones se encargan de filtrarla y eliminarla por medio de la orina. Paros respiratorios y cardiacos, espasmos vasculares, daños neurológicos e inestabilidad son las principales consecuencias de las lesiones por rayos (Torres, Rojas, 2003). 
Las investigaciones realizadas en las últimas décadas sobre lesiones y muertes relacionadas con fenómenos atmosféricos en el mundo, indican que los rayos han sido consistentemente la tercera causa de muerte y la segunda causa de lesiones, superada solamente por las inundaciones (Cooper, 2005).

El 24 de octubre de 2002 un rayo acabó con la vida de Herman el “Carepa” Gaviria y Giovanni Córdoba, dos recordados futbolistas de la selección colombiana de futbol; el 6 de octubre del año 2014 se presentó la muerte de 11 indígenas y 18 heridos de la comunidad Wiwa en la Sierra Nevada de Santa Marta, Colombia, noticias que reflejan el alto riesgo por rayos al que se enfrenta la población colombiana.

Según nuestros estudios estadísticos con datos del Departamento Administrativo Nacional de Estadística (DANE) y el Ejército Nacional, en Colombia los rayos producen 2 muertes por cada millón de habitantes; una cifra significativa si se compara con países como China, que tiene 0,3 muertes por millón, Malasia 0,8, Francia, 0,2, Brasil 0,8 o Estados Unidos con 0,3.

Además, las normas internacionales como la International Electrotechnical Commission (IEC) (IEC 62305-2 (Ed.3), 2013), contienen información basada en experiencias de países con bajo riesgo por rayos, muy diferentes a la colombiana y por ello se elaboró una Norma técnica colombiana NTC 4552.

\section{La historia y la literatura en la interpretación del rayo}

Si bien la ciencia moderna ofrece hoy en día la posibilidad de conocer y comprender los fenómenos de la naturaleza, la historia nos proporciona similares interpretaciones en otro lenguaje: el mítico. La figura 6 ilustra las diferentes interpretaciones míticas y religiosas que se le han dado al fenómeno del rayo en diferentes culturas a través de los siglos.

Un ejemplo de la interpretación de un mismo fenómeno en dos lenguajes diferentes lo encontramos en la creación de la vida en el universo, al comparar la creencia mítica de la ancestral y milenaria cultura Maya con el resultado científico llevado a cabo en nuestro tiempo en la Universidad de Cornell, Estados Unidos, según las cuales el rayo es el detonante de la vida en el universo (Torres, 2002).

Un análisis del significado de las invocaciones Mayas para la lluvia, muestra, por ejemplo, el conocimiento que ya tenían sobre la diferencia que actualmente, en lenguaje científico, se tiene entre rayos nube - tierra y entre nubes, así como las relaciones no directas que hoy en día conocemos entre número de días con rayos y número de días con lluvia.

Había meses y días especiales en que invocaban a los Chacs para la lluvia y en estas invocaciones utilizaban ciertos términos cuyo significado estaba relacionado con las lluvias. Por ejemplo: Lelemcaanchaac, “el dios de la lluvia
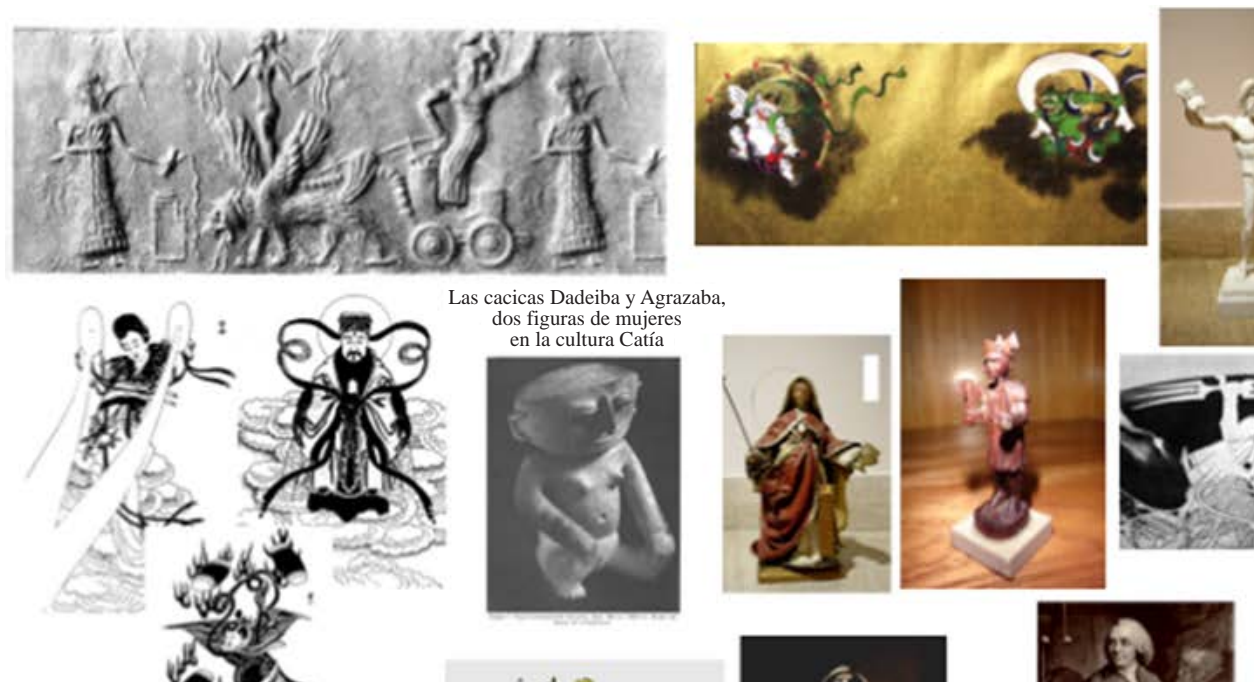

Las cacicas Dadeiba y Agraza dos figuras de mujeres
en la cultura Catía
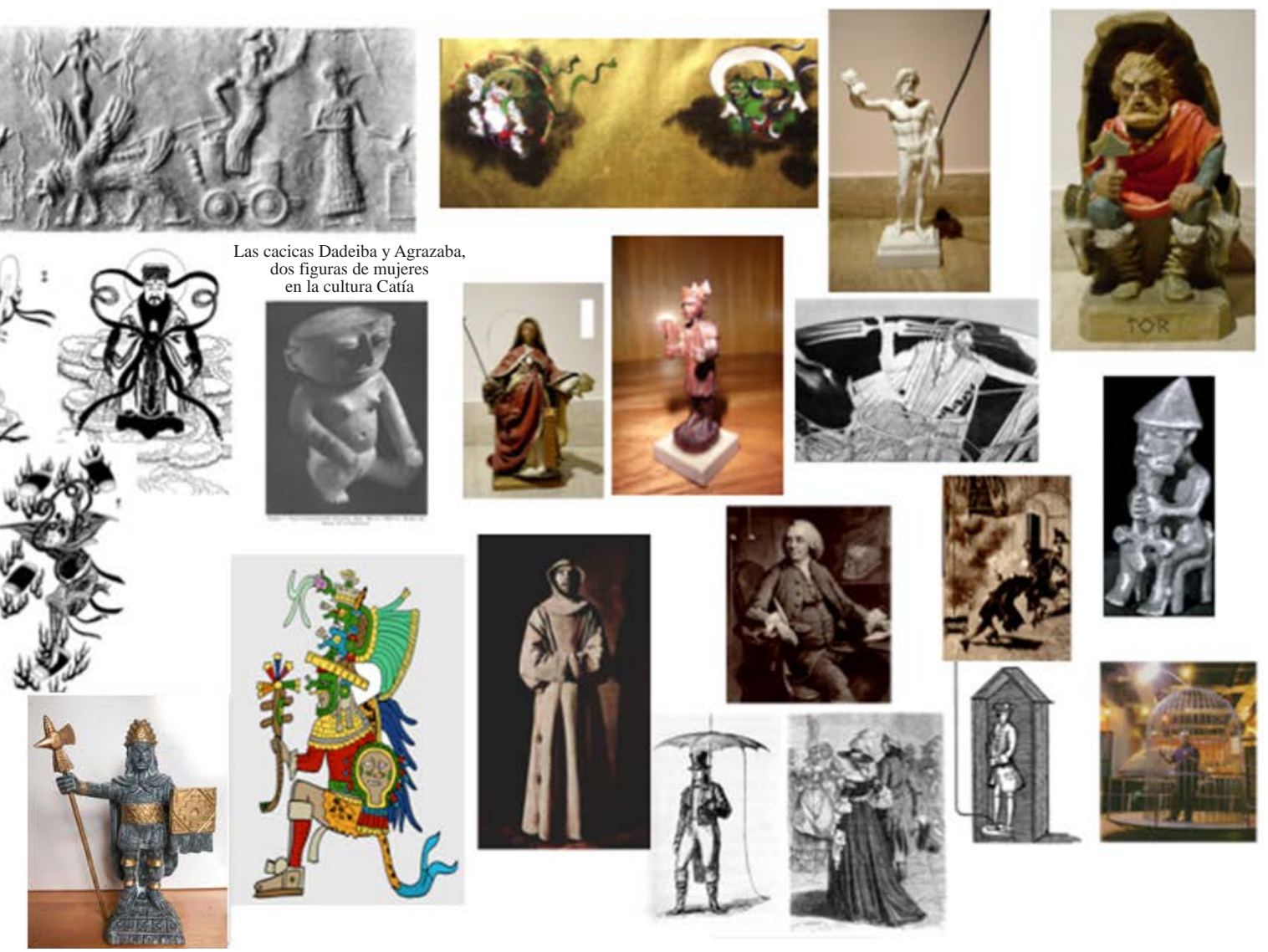

Figura 6. Interpretaciones del fenómeno del rayo en diferentes culturas a través del tiempo. 
látigo relampagueante...”, Mizencaanchaac, "rayo que barre el cielo...” y Lelencaanil, "tormenta de muchas nubes y poca agua.” (Torres, 2015).

Así mismo el rayo ha estado presente en las interpretaciones religiosas con la santa turca Santa Bárbara la patrona de las tormentas, cuyo día de celebración es el 4 de diciembre. Nació en Nicomedia, la capital de Bitinia, hoy día Ismidt en Turquía asiática, donde vivió y fue martirizada hacia el año 235 a la edad de 16 años. Fue canonizada por la iglesia católica en el año 1568 y nace así la leyenda de Santa Bárbara, patrona de los rayos (Torres, 2015).

Muchos sitios en Colombia y del mundo cristiano tienen el nombre de la santa patrona de los rayos, como el caso del barrio Santa Bárbara en el centro de la ciudad de Bogotá, debido a que, según cronistas de la época, allí impacto el primer rayo en la recién fundada ciudad de Santafé de Bogotá en el año 1565 (Torres, 2015).

El sincretismo religioso en la isla de Cuba llevo a Celina González y a Reutilio Domínguez a plasmar en una bella canción cubana la conciliación entre la santa cristiana y el dios africano del rayo Changó con la canción mundialmente conocida en las voces de Celina y Reutilio: "Santa Bárbara bendita... que viva Chango” (Torres, 2015).

El 4 de octubre la iglesia católica celebra la fiesta de San Francisco de Asís y, de acuerdo con la tradición oral, ese día, en los cerros orientales de la ciudad de Bogotá se presenta "el cordonazo de San Francisco", pues ese día San Francisco sacude el cordón de su hábito y castiga con cordonazos a los habitantes que han pecado. De acuerdo con los estudios que hemos realizado sobre rayos en la ciudad de Bogotá, en el mes de octubre se presenta la segunda temporada de rayos debido a que, meteorológicamente, se da el paso de la zona de confluencia intertropical por esta latitud y es el origen de este fenómeno, con mayor énfasis en los cerros orientales de la ciudad de Bogotá. La interpretación que le ha dado la iglesia católica sucede también en esta época en países tropicales como México, Venezuela, Panamá, parte de Brasil y África (Torres, 2015).

Pero la historia y la literatura también han descrito e interpretado el fenómeno del rayo. La parte media del Valle del Río Magdalena (o mejor conocido como Magdalena Medio) se caracteriza por la presencia de un sistema de alto nivel ceráunico, con valores promedio entre 120 y 160, en la región conocida como Catatumbo en zona limítrofe con Venezuela.

La más reciente publicación de resultados de medición satelital de la NASA (Albrecht, 2016) muestra, por ejemplo, que el sitio de mayor actividad de rayos del mundo estaría ubicado en la región del Catatumbo y específicamente en el lago de Maracaibo y de las 10 poblaciones de mayor actividad de rayos en la zona de Suramérica, 7 están ubicadas en Colombia. La actividad de rayos en esta región llevó a Alexander von Humboldt (Humboldt, 1956) a llamar este fenómeno como el "rayo del Catatumbo". El naturalista y explorador alemán lo describe como "explosiones eléctricas que son como fulgores fosforescentes...”, siendo reseñado luego por el geógrafo Agustín Codazzi como un "relámpago continuado que parece surgir del río Zulia y sus alrededores" (Codazzi, 1960).

Las observaciones de Humboldt son coincidentes con los resultados de la investigación de los últimos 30 años en zona tropical, fundamentalmente en Colombia Zavrostky, (1991), por ejemplo, en 1991 sugiere la hipótesis de rayos permanentes debido a colisión de corrientes de aires fríocaliente. No se descarta al uranio como agente co-causal. Este fenómeno se caracteriza por ser un relámpago casi continuado y silente, que se produce en nubes de gran desarrollo vertical formando arcos eléctricos entre los 2 y un poco más de 10 kilómetros de altura, a medida que los vientos alisios penetran en la superficie del lago de Maracaibo en horas de la tarde, cuando la evaporación es mayor y ascienden por el sistema montañoso de la Serranía del Perijá (de 3.750 msnm) en Colombia y la Cordillera de Mérida, el ramal venezolano de los Andes (de $5.000 \mathrm{msnm}$, aproximadamente).

Anecdóticamente el rayo del Catatumbo frustró en 1595 un ataque del pirata inglés Francis Drake a la ciudad de Maracaibo y fue narrado en un bello episodio en la obra épica "La Dragontea” (Lope de Vega, 1598), y relata cómo la luminosidad desplegada por el relámpago le permitió a un vigía divisar las naves de Drake y alertar a la guarnición, que logró repeler el ataque nocturno.

Además, durante la guerra de independencia, el rayo del Catatumbo sirvió de faro para la fuerza naval del Almirante neogranadino Jose Prudencio Padilla, quien logró derrotar a los navíos españoles el 24 de julio de 1823 en la batalla naval del lago de Maracaibo.

\section{La química y el fenómeno del rayo}

Aunque, generalmente, se tiene el prejuicio de que los rayos son un fenómeno atmosférico negativo relacionado con muerte y destrucción, que llega a producir brontofobia o ceraunofobia, un miedo persistente, anormal e injustificado a los rayos, ideas que no son totalmente ciertas y por tanto injustificadas, pues los rayos también son fundamentales en la vida sobre la tierra (Torres, 2015).

Si bien es un fenómeno natural que puede experimentar temor, los rayos, cuando fijan el nitrógeno al impactar en el suelo, son un verdadero banquete para las plantas, que se refleja rápidamente en un vigor y crecimiento extraordinario. El ciclo del nitrógeno es cada uno de los procesos biológicos y abióticos, es decir, que no forma parte o no es producto de los seres vivos, en que se basa el suministro de este elemento a los seres vivos.

El primer paso en el ciclo es la fijación del nitrógeno atmosférico $\left(\mathrm{N}_{2}\right)$ a formas distintas susceptibles de incorporarse a la composición del suelo o de los seres vivos, como el ion amonio $\left(\mathrm{NH}_{4}+\right)$ o los iones nitrito $\left(\mathrm{NO}_{2}-\right)$ o nitrato $\left(\mathrm{NO}_{3}-\right)$. Aunque el amonio puede ser usado por la mayoría de los organismos vivos, las bacterias del suelo derivan la energía de la oxidación de dicho compuesto a 
nitrito y finalmente a nitrato. El nitrógeno en forma de $\mathrm{N}_{2}$, se encuentra formando parte de la atmósfera, integrando un $78 \%$ de la misma. Es un elemento bastante inerte, no reacciona fácilmente con otros compuestos, así que antes de ser aprovechado por la mayoría de los seres vivos, debe pasar por un proceso de fijación. Este proceso de fijación de nitrógeno está definido como su combinación con oxígeno o hidrógeno. La combinación es llevada a cabo por varias vías distintas. La fijación natural puede ocurrir por procesos químicos espontáneos, como la oxidación que se produce por la acción de los rayos, que forma óxidos de nitrógeno a partir del nitrógeno atmosférico. En la estimación de la producción mundial de NOx por rayos, el parámetro que más influye es la longitud del canal de descarga dentro de la nube. Para la longitud total de canales dentro de la nube de un rayo típico nube-tierra de alrededor de $45 \mathrm{~km}$, se estima que la producción mundial anual de NOx es de aproximadamente 4Tg.

Adicionalmente, el adelgazamiento de la capa de ozono puede tener consecuencias graves para la vida en la tierra. Los rayos ultravioletas (UV) pueden destruir el ADN, moléculas que se necesitan para la reproducción de los seres vivos. Los rayos ultravioletas alteran los procesos fisiológicos y de desarrollo e indirectamente modifican su forma, distribución de los nutrientes y etapas de crecimiento lo cual es perjudicial para su supervivencia. De acuerdo con estudios sobre la capa de ozono, el ozono troposférico en mayor porcentaje es formado por reacciones químicas a partir de los óxidos de nitrógeno producidos por los rayos.

\section{La innovación tecnológica}

La innovación tecnológica como resultado inherente a la investigación, lleva implícito un largo proceso de apropiación, construcción y generación de conocimiento que se conoce como investigación tecnológica y que ha sido descrita en los apartados anteriores. Y este proceso requiere del sustrato académico de las ciencias básicas que, en definitiva, puede llevar a aplicaciones empresariales con registros y patentes.

Con base en los conocimientos logrados sobre la física del rayo, los modelos matemáticos y mediante tesis de pregrado, maestría y doctorado nacionales (Aranguren, 2011) fue posible, con un medidor de campo eléctrico modificado, lograr los primeros resultados de nuevo conocimiento que permitieron predecir, por primera vez en el mundo, el impacto de un rayo. En condiciones de buen tiempo el campo eléctrico ambiental es de aproximadamente $120 \mathrm{~V} / \mathrm{m}$. Las nubes de tormenta se caracterizan por generar variaciones de campo eléctrico alcanzando niveles de hasta $15 \mathrm{kV} / \mathrm{m}$ en zonas planas. En las mediciones locales realizadas en un trabajo de doctorado (Aranguren, 2011) en la Universidad Nacional de Colombia, observamos que se registraban cambios en diferentes parámetros derivados de la señal de campo eléctrico. Estos efectos fueron analizados y se pensó en que podrían ser debidos a ruidos en la medición.
Se propuso, entonces, realizar mediciones en otros sitios de Colombia y en Europa, gracias a los contactos con colegas que investigan el fenómeno.

Un análisis de las mediciones locales en Colombia y Europa, así como datos de mediciones en la estación de la NASA en Cabo Cañaveral, dieron como resultado una explicación física, cuyos resultados fueron los primeros criterios para predecir la ocurrencia de rayos dentro de una región de interés, a través de la activación de una alarma temprana. Este proceso es lo que se conoce en idioma ingles como "serendipity", es decir, un hallazgo afortunado e inesperado que se produjo cuando se buscaba otra cosa distinta. El área de predicción se definió dentro del rango de alcance que tiene el sensor de campo eléctrico, el cual puede ser hasta $20 \mathrm{~km}$ de radio (Torres, 2014).

Trabajos con nuevas tesis de maestría y doctorado (Soto, 2015, Inampues, 2014, López, 2011), Aranguren, 2011), permitieron profundizar en la predicción de rayos logrando definir el tiempo de predicción en aproximadamente media hora con una eficiencia de más de $90 \%$.

De estos resultados de investigación nació una empresa de innovación tecnológica, que en tecnología se conoce como una empresa "spin-off". Esta empresa colombiana ha venido encontrado innovaciones tecnológicas, con patentes y aplicaciones para la protección contra rayos de personas, de sistemas y equipos, que se ha extendido a una gran variedad de actividades que incluyen, además de la protección de vidas de personas en espacios abiertos (parques, escenarios deportivos, lugares de trabajo y otros), la protección de aeropuertos, sistemas de distribución y transporte de energía eléctrica, minas de extracción en campo abierto, campos petroleros, entre muchos otros.

\section{Recursos gerenciales: gestión de riesgo por rayos}

Debido a que la sociedad moderna es cada día más dependiente de los equipos de cómputo, del servicio de energía eléctrica, de los sistemas de comunicación y, en general, de los equipos eléctricos y electrónicos, la ingeniería colombiana debe responder con diseños adecuados y protecciones eficaces de estos contra las perturbaciones electromagnéticas, como las producidas por un rayo, que son, en magnitud, las más altas del mundo según resultados de investigación realizados en Colombia. Para ello es necesario incorporar el análisis del fenómeno del rayo a la gestión del riesgo (Torres, 2010).

La gestión de riesgo ha sido desarrollada fundamentalmente en las áreas de la economía y, recientemente se ha enfocado en el tema de desastres, entre los cuales está el fenómeno del rayo. Es un enfoque estructurado para manejar la incertidumbre relativa a la amenaza de un rayo, a través de una secuencia de actividades humanas que incluyen la evaluación de riesgo por rayos, estrategias de desarrollo para manejarlo y mitigación del riesgo utilizando recursos gerenciales. Las estrategias incluyen fundamentalmente mitigar los efectos negativos del riesgo por rayos. 
La gestión de riesgo por rayos la hemos desarrollado mediante una tecnología que se ha bautizado como 3P y se refiere a la Predicción, Protección y Prevención de rayos con innovaciones tecnológicas, aplicaciones y tecnologías para la protección de personas, de sistemas y equipos para la gestión de riesgo por rayos.

La primera P, de predicción, se logra mediante el molino de campo eléctrico (bautizado como PreThor) al que se le modificó el hardware y se hicieron modificaciones en la medición de la polaridad (carga eléctrica positiva y negativa de las nubes). La figura 7 presenta esquemáticamente como se logra la predicción con el molino de campo para un sitio determinado.

Son dos placas paralelas, una de ellas en movimiento, separadas por un aislamiento que contienen un circuito unido a un osciloscopio (instrumento de visualización electrónico que representa gráficamente señales eléctricas) y que permite visualizar las señales positivas o negativas. Al medir el campo eléctrico atmosférico (electricidad en la atmosfera), se da un aumento de la señal mientras se genera una concentración de carga en las nubes, que llega hasta un punto de saturación que marca el umbral en el cual se obtienen las variaciones que detecta el equipo (Aranguren, 2009).

La otra P se refiere a la protección, cómo proteger espacios concurridos al aire libre. Existen formas para lograr esto mediante la utilización de jaulas de Faraday, una caja metálica que protege de los rayos, conduciéndolo efectivamente a tierra, similar al montado en el centro interactivo Maloka de Bogotá, Figura 8. Con este módulo instalado en Maloka se logra la apropiación social del fenómeno.

Por último, la prevención, se puede generar a través de cartillas y capacitaciones que guíen a las personas que trabajan, hacen deporte o asisten a espectáculos multitudinarios al aire libre. En este punto es fundamental integrar los conceptos de psicología social, para lograr que las comunidades entiendan y asuman la responsabilidad cual debe ser el comportamiento individual y colectivo ante el fenómeno del rayo.

\section{Trabajos futuros en la investigación sobre el rayo y el cambio climático global}

Aunque ha habido intentos investigativos de caracterizar la fenomenología del rayo, su origen puede ser atribuible a cinco factores que influencian su actividad en una zona determinada: vientos, orografía, superficie, subsuelo y cambios por actividad humana los cuales serán fundamentales en las próximas actividades de investigación sobre rayos y requerirán de disciplinas como la geología y la geografía.

Vientos. La actividad de rayos permanentes es ocasionada por la circulación de sistemas de vientos, cuyas nubes de tormenta transportan cargas eléctricas en su interior. En el caso colombiano estos vientos son los alisios del Noreste y del Sureste que al confluir forman la Zona de Confluencia Intertropical ZCIT y son la materia prima para la generación de rayos.

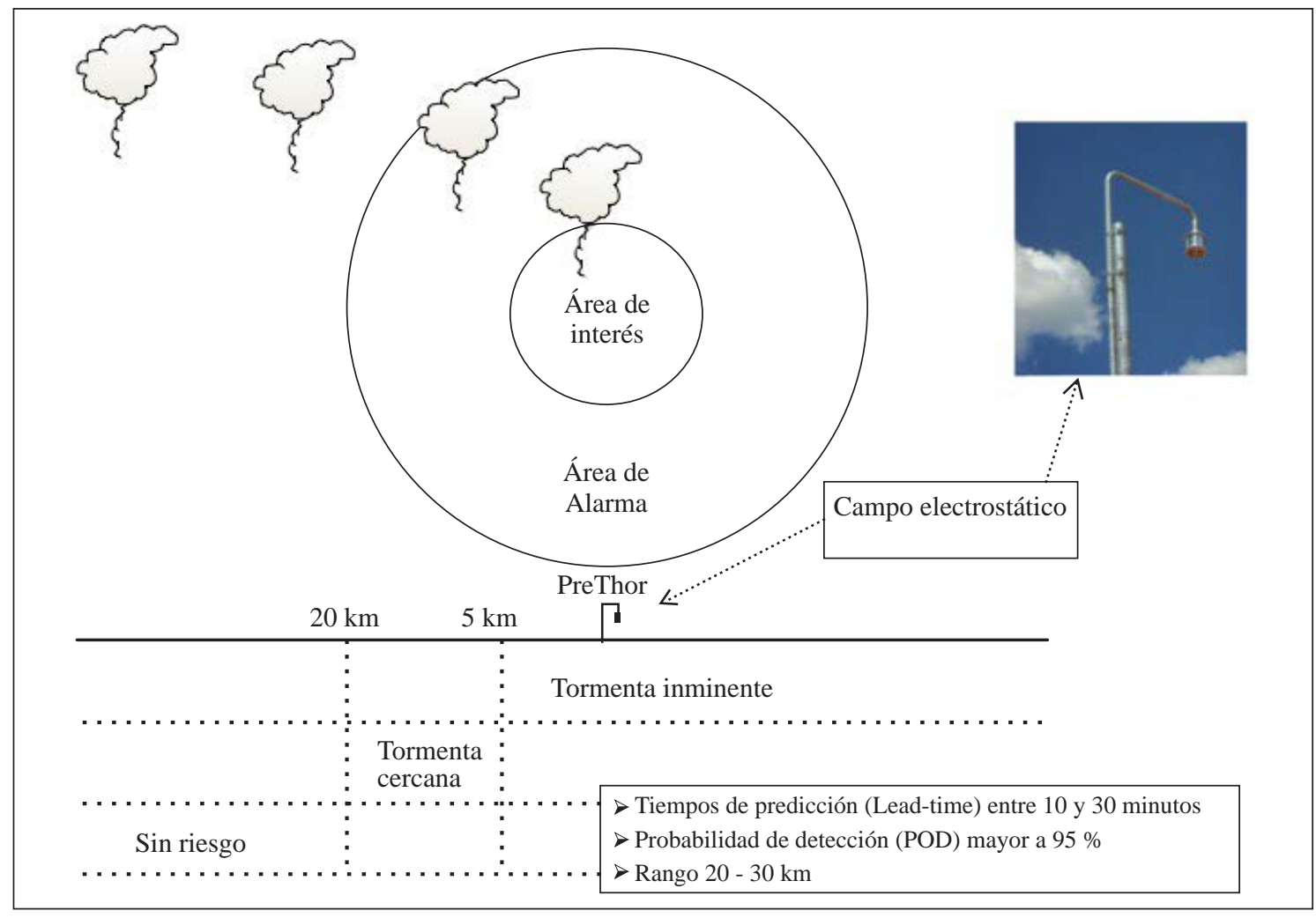

Figura 7. Procesamiento del campo eléctrico ambiental por el molino PreThor. 


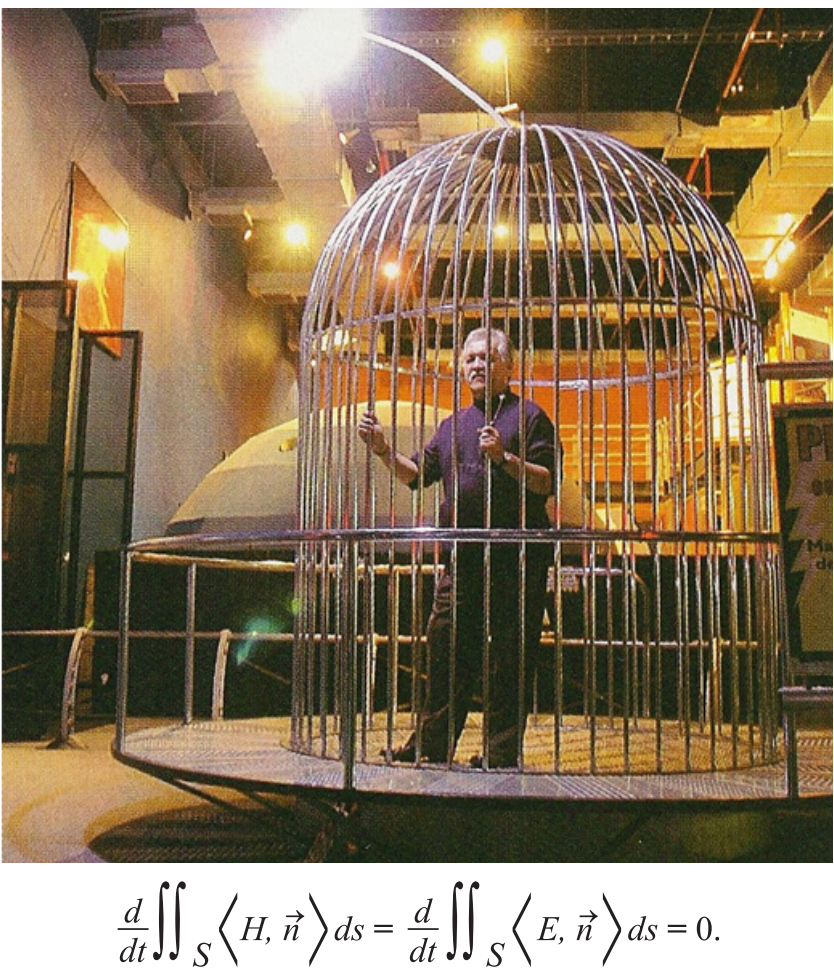

Figura 8. Protección Externa: Jaula de Faraday en el centro Maloka, Bogotá.

Orografía. El sistema montañoso de la región que interactúa con el sistema de vientos, produciendo concentración de nubes de tormenta y facilidad para generar rayos por el fenómeno de convección. Un claro ejemplo de este factor se da en la ciudad de Bogotá, cuando en época de paso de la ZCIT, los vientos alisios llevan nubes de tormenta que chocan contra las montañas orientales y por convección se da la actividad de rayos después del mediodía.

Superficie. La diferencia de la actividad de rayos entre la tierra y el océano se atribuye generalmente al contraste en las propiedades físicas de cada superficie, pero el logro de una explicación cuantitativa acertada ha tomado algún tiempo (Williams, et. al, 2002). La superficie está ligada a otro factor que tiene que ver con los cambios por actividad humana.

Subsuelo. La posibilidad de la existencia de algún agente causal en el subsuelo, por ejemplo, fuentes geomagnéticas o radiactivas que pueden producir cargas iónicas en el entorno; la región del Catatumbo, por ejemplo, es rica en uranio y, en general, se tiene que las mayores zonas de actividad de rayos presentan una correlación observacional con materiales metálicos como hierro, oro, níquel, plata, etc.

Cambios por actividad humana. Se refiere al termino de efecto isla por calentamiento urbano, que en idioma ingles se conoce como el efecto Urban Heat Island (UHI). El efecto isla por calentamiento urbano en un área metropolitana es significativamente más caliente que sus alrededores debido a las actividades humanas (Cole, et. al., 2016). A medida que la población crece y hay mayor actividad humana, tiende a ampliar su área y aumentar su temperatura media. El término UHI se refiere, entonces, a cualquier área que es consistentemente más caliente que sus alrededores. Este efecto ha sido estudiado para analizar el aumento de la actividad de rayos, por ejemplo, en la población de Manaos, Brasil y su área selvática alrededor (Pinto, et al, 2014).

En estudios sobre actividad de rayos en zonas petroleras en Colombia hemos observado que a medida que aumenta la actividad humana en la zona, por ejemplo, caminos polvorientos que levantan partículas de tierra hacia la atmosfera, aumenta la actividad de rayos. Estos temas valen la pena analizarlos a mayor profundidad para encontrar respuestas a estos incrementos.

Este argumento hipotético con sus 5 factores es válido para cualquier análisis de actividad de rayos en una zona determinada y merece un estudio investigativo más a profundidad. Estudios recientes en física atmosférica estiman que el calentamiento global incrementará significativamente la actividad de rayos. El estudio basado en la información de la Red Nacional de Detección de Rayos de Estados Unidos (NLDN) estima que por cada dos rayos que cayeron en al año 2000, caerán tres en 2100 y este aumento podría tener consecuencias desastrosas. El estudio del investigador David Romps, de la Universidad de California, Berkeley (Romps, et. al, 2014), indica que por cada grado centígrado que aumente la temperatura global, la frecuencia de los rayos se incrementará un $12 \%$. Además de provocar más incendios naturales, las constantes descargas eléctricas atmosféricas alterarán la composición química de la atmósfera. Conforme el planeta se calienta, habrá más energía para producir rayos más potentes. Se estima que, durante el siglo XXI, la tierra se calentará $4{ }^{\circ} \mathrm{C}$.

La relación rayos - cambio climático es un tema de investigación que requiere un trabajo científico de fondo, pues los primeros resultados de Romps han sido realizados únicamente para los Estados Unidos, pero no en la zona tropical.

Por estas razones, un objetivo que vale la pena en la investigación del cambio climático es la identificación de parámetros físicos mensurables, los cuales son dependientes, no linealmente, de los cambios en la temperatura atmosférica; de esta manera se podría tener alguna ganancia en la medición del calentamiento global. El potencial ionosférico - PI es una de estas variables y está asociado con la electrificación de las nubes, las cuales a su vez están controladas por fluctuaciones del equilibrio hidrostático, que es causado por diferencias de temperatura del orden de unos pocos grados Celsius (Torres, 2002).

La convección profunda (Deep Convection) (cuando el aire húmedo se eleva en una atmósfera potencialmente inestable, hay una energía potencial convectiva disponible (CAPE por sus siglas en inglés) para que éste suba su nivel de fluctuación neutra, usualmente a algún lugar de la troposfera superior. Esta elevación y la atmósfera inestable dan como resultado la formación de nubes, precipitación y células convectivas de elevación profunda) es de gran 
importancia tanto en la generación de carga para el circuito eléctrico global como en la generación de calor para la celda de Hadley, se origina en el fenómeno de inestabilidad condicional del aire. La fuente de energía necesaria para el desarrollo de este proceso es CAPE, una cantidad primero conceptualizada por Margules en 1905, pero definida por Moncrieff y Miller en 1976 (Torres, 2002).

Los experimentos de campo realizados por Williams en Darwin (Australia) en 1990, han demostrado una sensitiva dependencia de la actividad de los rayos con la temperatura de bulbo húmedo, medida sobre la superficie y la CAPE (Williams, Stanfill, 2002). El análisis de las mediciones de temperatura de bulbo húmedo y actividad de rayos nube - tierra e intra nubes muestran que en la estación seca en Darwin (mayo - octubre) es menor que el valor umbral y por consiguiente no se observan ni convección profunda ni actividad de rayos (Torres, 2002).

\section{Conclusiones}

- La principal conclusión de este artículo es exponer con un ejemplo práctico y concreto la metodología y estrategia para desarrollar ciencia, tecnología e innovación en un país como Colombia, como un proceso de largo plazo de apropiación, construcción y creación autónoma de conocimiento alrededor de una problemática propia de su entorno con categoría conceptual de un trabajo interdisciplinario.

- Hoy en día la interdisciplinariedad respecto al fenómeno del rayo ofrece un marco metodológico que a futuro debe estar basado en la intersección de las ciencias básicas como la física, las matemáticas, la química, las ciencias biológicas, la historia, la literatura, las teorías en psicología y otras que pueden llegar a necesitarse, si se desea realizar un trabajo investigativo creativo e innovador.

- La interdisciplinariedad es fundamental al hablar del desarrollo científico del rayo, porque para poder comprender a profundidad el fenómeno y proponer soluciones tecnológicas e innovaciones, es indispensable la interacción entre aquellas disciplinas que le son afines para aportar al conocimiento del fenómeno en el concierto mundial.

- A futuro, la relación rayos - cambio climático es un tema de investigación que requiere un trabajo científico de fondo, pues los primeros resultados recientemente han sido efectuados únicamente para los Estados Unidos, pero no en la zona tropical.

- Vientos, orografía, superficie, subsuelo y cambios por actividad humana serán aspectos fundamentales en las próximas actividades de investigación sobre rayos y requerirán de disciplinas como la geología y la geografía.

- La innovación es inherente a la investigación científica que requiere como fundamento las ciencias básicas. Para ello se requieren un proceso de décadas con un principio básico: en el método científico no existen verdades absolutas, sino certezas temporales y los resultados tienen que ajustarse continuamente.

\section{Conflicto de intereses}

El autor declara no tener conflicto de intereses.

\section{Referencias}

Agoris, D., Charalambakos, V., Pyrgloti, E., Grzybowski, S. (2004). A computacional approach on study of Franklin rod height impact on striking distance using a stochastic model. J. Electrostatics. 60: 175-181.

Albrecht, R. (2016). Where are the lightning hotspots on Earth? AMS.

Anderson, R., Eriksson, A. (1980). Lightning parameters for engineering application. Electra 69: 65-102.

Aranguren, D. (2011). Desempeño de sensores de campo electrostático en sistemas de alerta de tormentas”, tesis de doctorado, Universidad Nacional de Colombia, Bogotá.

Aranguren, D., Montanyà, J., Torres, H., Solà, G., March, V., Romero, D. (2009). On the lightning hazard warning using electrostatic field: analysis of summer thunderstorms in Spain. J. Electrostatic 67: 507-512.

Aranguren, D., Lopez, J., Inampues, J.C., Torres, H., Betz, H. (2016). Cloud-to-ground lightning activity in Colombia and the influence of topography. Journal of Atmospheric and Solar-Terrestrial Physics, 154: 182-189.

Berger, K., Anderson, R B., Kroninger, H. (1975). "Parameters of lightning flashes". Cigre Electra, 41: 23-37.

Codazzi, A. (1960). Resumen de la geografía de Venezuela. Paris, 1841. Imprenta Fournier, Bancroft Library.

Conti, E., Perez, E., Soto, F., Silveira, S., Visacro, H. Torres, H. (2010). Calculation of Lightning-Induced Voltages on Overhead Distribution Lines Including Insulation Breakdown. Journal IEEE Transactions on Power Delivery, 25: 3078-3084.

Cole, P., Krehbiel, T.J., Henebry, G.M. (2016). Web-Enabled Landsat Data Time Series for Monitoring Urban Heat Island Impacts on Land Surface Phenology. IEEE Journal Selected Topics in Applied Earth Observations and Remote Sensing, 9: 2043-2050.

Cooper, M.A., Holle, R.L., Andrews, Ch. (2010). Distribution of lightning injury mechanism. Proceeding 30th International Conference on Lightning Protection (ICLP). doi: 10.1109/ ICLP.2010.7845948, pp. 1- 4.

Cooray, V. (2003). The lightning flash. Institution of Electrical Engineers. London, UK.

El Popol Vuh. (1975) Ed. Ariel, pp. 12-14.

Herrera, J. (2006). Nuevas aproximaciones en el cálculo de tensiones inducidas por descargas eléctricas atmosféricas. Tesis de doctorado, Universidad Nacional de Colombia, Bogotá.

Humboldt, A., Bonpland, A. (1956). Viaje a las regiones equinocciales del Nuevo Continente. Tomo III, pp 226. Traducción de Lisandro Alvarado.

IEC Standard 62305-2(Ed.3) on Protection against lightning, 2013.

Inampues, J.C. (2014). Predicción contra rayos y redes inteligentes. Tesis MSc., Universidad Nacional de Colombia - Bogotá.

Lee, L.C., Lim, K., Meiappa, M., Liew, A. C. (1979). Determination of Lightning Current Using Frame Aerials. IEEE Transactions on Power Apparatus and Systems, 98: 16691675. doi: 10.1109/TPAS.1979.319485

Lope de Vega y Carpio. (1598). La Dragontea, al Príncipe nuestro Señor, en Valencia por Pedro Patricio Mev. Ayuntamiento de Madrid, España. 
López, J. (2011). Metodología para predicción de tormentas eléctricas a partir de mediciones de campo electrostático ambiental y sistemas de localización de rayos en zona montañosa”, MSc tesis, UN Manizales.

Lupo, G., Petrarca, C., Tucci, V., Vitelli, M. (2000). EM fields associated with lightning channels: on the effect of tortuosity and branching" IEEE Transactions on Electromagnetic Compatibility. 42: 394- 404.

MacGorman, D., Rust, W. (1998). The Electrical Nature of Storms. Oxford University Press. New York, New York.

Norma Técnica Nacional. NTC 4552 (2008). Protección contra descargas eléctricas atmosféricas, ICONTEC, Bogotá.

Pérez, E. (2006). Avances en el modelamiento y experimentación de tensiones inducidas en redes de distribución. Tesis de doctorado, Universidad Nacional de Colombia, Bogotá.

Petrov, N., Petrova, G., D’Alessandro, F. (2003). Quantification of the probability of lightning strikes to structures using a fractal approach. IEEE Transactions on Dielectrics and Electrical Insulation, 10: 641- 654.

Pinto, O. (2013). Lightning and climate: A review. Proceeding International Symposium on Lightning Protection (XII SIPDA). doi: 10.1109/SIPDA.2013.6729250. pp. 402-404.

Rakov, V., Uman, M. (2003). Lightning: physics and effects" Cambridge University Press. Cambridge, UK.

Rakov, V.A. (1992). Data acquired with the LLP lightning locating systems. Meteor. Gidrol. 7: 105-114.

Romps, D.M., Seeley, J.T., Vollaro, D., Molinari, J. (2014). Projected increase in lightning strikes in the United States due to global warming. Science 346: 851-854. doi: 10.1126/ science.1259100.

Sagan, C. (1985). Cosmos, Ed. Planeta, p. 38.

Silveira, F. Visacro, S., Herrera, J., Torres, H. (2008). Evaluation of Lightning-Induced Voltages over Lossy Ground by the Hybrid Electromagnetic Model. Trans Electromagnetic Compatibility IEEE, TEMC-130-2008.R1.

Soto, E. (2015). Lightning induced voltages on overhead distribution networks placed over non-flat terrains. Tesis doctoral, UN Manizales.

Torres, H. (1998). Espacio y tiempo en los parámetros del rayo, ensayo sobre una hipótesis de investigación. Trabajo de investigación presentado a la Universidad Nacional de Colombia para promoción a la categoría de Profesor Titular. Bogotá.

Torres, H. (2010) ¿Qué rayos sabemos? Rev. Acad. Colomb. Cienc. Ex. Fis. Nat., 34 (131): 193-208.

Torres, H. (2015). El rayo en el trópico. Colección apuntes maestros, Ed. UN, Bogotá, Colombia.
Torres, H. (2002). El Rayo, Mito, Ciencia y Tecnología. Unibiblos, Bogotá, 2002.

Torres, H. (2010). Protección contra rayos - 2a Edición. Editorial ICONTEC, Universidad Nacional de Colombia, Bogotá.

Torres, H. (2014). ¿Qué rayos sabemos sobre innovación tecnológica? Ed. Orvisa, Bogotá, Colombia.

Torres, H., Perez, E., Younes, C., Aranguren, D., Montaña, J., Herrera, J. (2015). Contribution to lightning parameters study based on some American Tropical Regions observations. IEEE Journal of Selected Topics in Applied Earth Observations and Remote Sensing, 8: 4086-4093. doi: 10.1109/JSTARS.2015.2428217

Torres, H., Rojas, J. (2003). Los rayos, efectos biológicos y prevención. Revista Innovación y Ciencia, 11: 32-40.

Torres, H., Rondon, D., Briceño, W., Barreto, L. (1996). Lightning peak current estimation analysis from field measurements in tropical zones. Proceedings 23rd. ICLP, Florence, Italy, Sep.

Vargas, M. (2006). Nuevo modelo integral del Canal de la Descarga Eléctrica Atmosférica y su enlace con estructuras en tierra”, Tesis de doctorado, Universidad Nacional de Colombia - Bogotá.

Vargas, M., Torres, H. (2008). Lightning leader model for straight, tortuous or branched channels - Part I: Model description. J. Electrostatic, 66: 482-488.

Vargas, M., Torres, H. (2008). Lightning leader model for straight, tortuous or branched channels - Part II: Model results. J. Electrostatic, 66: 489-495.

Visacro, S., Soares, A. (2004). Statistical analysis of lightning current parameters: Measurements at Morro do Cachimbo station. Journal of Geophysical Research, 109, D01105, doi: 10.1029/2003JD003662

Whipple, F.J.W. (1929). On the association of the diurnal variation of electric potential in the weather with the distribution of thunderstorms over the globe. Quart. J. Roy. Met. Soc., 55: 1-17.

Williams, E., Stanfill, S. (2002). The physical origin of the landocean contrast in lightning activity. Comptes RendusPhysique, 3: 1277-1292, 2002.

Wilson, C. T. R. (1920). Investigations on lightning discharges and on the electric field of thunderstorm. Philos. Trans. A., 221: 73-117.

Younes, C. (2006). Metodologías para la correlación de parámetros del rayo con características geográficas y meteorológicas. Caso colombiano, tesis de doctorado, Universidad Nacional de Colombia, Bogotá, 2006.

Zavrostky, A. (1991). Carta Ecológica. Ediciones ULA № 56. Mérida, Venezuela. 